\title{
AJUSTE DE MODELOS MATEMÁTICOS PARA ESTIMAR BIOMASSA AÉREA EM FLORESTA OMBRÓFILA DENSA
}

\author{
Péricles Silveira ${ }^{1}$ \\ ${ }^{1}$ Eng. Florestal, Dr., Curitiba, PR, Brasil - pericles.silveira@dprf.gov.br \\ Recebido para publicação: 25/07/2007 - Aceito para publicação: 04/12/2008
}

\begin{abstract}
Resumo
Este trabalho teve como objetivo testar modelos matemáticos, 10 aritméticos e 9 logarítmicos, que visam estimar biomassa individual aérea em Floresta Ombrófila Densa, e escolher o melhor deles, com base nos critérios estatísticos mais comumente utilizados na ciência florestal. A área de estudo está localizada nos municípios de Presidente Nereu Ramos e Apiúna, no estado de Santa Catarina. A base de dados para este estudo é proveniente de uma pesquisa sobre inventário florestal que tem como um dos objetivos a quantificação da biomassa em propriedades da Companhia Hemmer Indústria e Comércio. Deste inventário, foram amostradas, pelo método destrutivo, 213 árvores, nas quais, para esta finalidade, foram medidos o diâmetro à altura do peito (DAP) e a altura total, como também foram realizadas as determinações da biomassa total acima do solo. Com base em uma matriz de correlação, foi realizada a seleção das variáveis com as melhores correlações para serem utilizadas nos modelos testados. O coeficiente de determinação ajustado $\mathrm{R}^{2}$ aj, erro padrão da estimativa (Syx\%), análise gráfica dos resíduos e amplitude residual (AR) foram os critérios utilizados para avaliar os modelos testados. De um modo geral, todos os modelos testados apresentaram bons ajustes, porém o modelo logarítmico, $\ln B T=b_{0}+b_{1} \ln d a p+b_{2} \ln h t$, com um $\mathrm{R}^{2}$ aj de 0,91 , Syx $\%$ de 26,37, AR de 78,25 e com base na análise gráfica de resíduos, foi o selecionado para estimar a biomassa total.

Palavras chave: Biomassa acima do solo; equações para biomassa; regressão; correlação; critérios estatísticos.
\end{abstract}

\begin{abstract}
Adjustment of mathematical models for estimating above-graound biomassin in a Dense Ombrophilous Forest. The aim of this work is to test mathematical models, 10 arithimatic and 9 logarithimic, intending to estimate individual above ground biomass in Dense Ombrophilous Forest. After it, and based on the most commonly used statistical criteria in forest science, the best model was chosen. The studied area is located in the municipalities of President Ramos and Apuína Nereu, in the State of Santa Catarina. The database for this study originally came from a research concerning the forest inventory that has as one of its aims the quantification of the biomass in the properties of the Hemmer Industry and Trade Company. From this inventory, 213 trees were sampled by the destructive method. Also, their diameters at breast height and total height were measured; the total biomass above ground was determined. Based on a correlation matrix, it was made a selection of the variables with the best correlation to be used in the tested models. The coefficient of adjusted determination $\mathrm{R}^{2}$ aj, standard error of estimate (Syx\%), graphic analysis of the residual waste and residual amplitude (RA) represented the criteria used to evaluate the tested models. Generally, all the tested models presented satisfactory adjustments, but the logarithimic model, $\ln B T=b_{0}+b_{1} \ln d a p+$ $b_{2} \ln h t$, with $\mathrm{R}^{2}$ aj of 0,91 , Syx $\%$ of 26,37 , AR of 78,25 and based on the graphic analysis of the residuals, was selected to estimate total biomass.
\end{abstract}

Keywords: Above-ground biomass; biomass equations; regression; correlation; statistical criteria.

\section{INTRODUÇÃO}

O volume de biomassa florestal e sua distribuição são fatores controladores do estoque de carbono global, servindo também como base para a predição futura da mudança climática (SEDJO, 1992; DIXON et al., 1994). Uma estimativa acurada da biomassa florestal e seu padrão de mudança no tempo é 
um pré-requisito para ajudar a entender a grande controvérsia sobre a função das florestas no ciclo do carbono (SEDJO, 1992; FAN et al., 1998; BROWN; SCHOEDER; KERN, 1999).

Por serem as florestas os maiores acumuladores de biomassa do planeta, a sociedade civil organizada e a mídia vêm demandando dos profissionais que atuam na área florestal a geração de informações e conhecimentos que possam auxiliar na redução dos riscos ambientais que se colocam diante da espécie humana (SANQUETTA, 2002). Um dos aspectos mais relevantes nos estudos de fixação de carbono em florestas é a variável biomassa, a qual precisa ser determinada e estimada de forma fidedigna, caso contrário não haverá consistência na quantificação do carbono fixado nos ecossistemas florestais (SANQUETTA, 2002).

Para Higuchi; Carvalho Júnior (1994), os estudos para quantificação de biomassa florestal dividem-se em métodos diretos (ou determinação) e métodos indiretos (ou estimativas). Determinação significa uma medição real feita diretamente da biomassa, por exemplo, a pesagem de um fuste inteiro por meio de um dinamômetro ou uma balança. Todas as árvores de uma determinada parcela são derrubadas e pesadas, sendo feita em seguida a extrapolação da avaliação amostrada para a área total de interesse. A estimativa de biomassa aérea pelo método indireto consiste em correlacioná-la com alguma variável de fácil obtenção e que não requeira a destruição do material vegetal. As estimativas podem ser feitas por meio de relações quantitativas ou matemáticas, como razões ou regressões de dados provenientes de inventários florestais (dap, altura e volume) ou por dados de sensoriamento remoto (imagens de satélite).

Somogyi et al. (2006) afirmam que avaliações de biomassa de forma indireta podem ser feitas por dois métodos, quando se trabalha em campo: um deles é usar dados de volume de árvores ou talhões e multiplicá-los por um fator ou fatores apropriados, denominados fatores de biomassa (BF), que convertem (expandem ou reduzem) as estimativas de volume para estimativas de biomassa, sendo escrito da seguinte forma:

$$
B=P \cdot B F
$$

Em que:

$B=$ biomassa em $\mathrm{kg}$ ou ton;

$P=$ variável de uma árvore ou talhão, por exemplo, volume $\mathrm{em} \mathrm{m}^{3}$;

$B F=$ fator apropriado da biomassa utilizado na conversão.

Outra forma de se estimar biomassa de forma indireta é realizando o ajuste de equações pelo uso de técnicas de regressão. Segundo Koehler; Watzlawick; Kirchner (2002), esse é o procedimento mais comum, em que algumas árvores são amostradas e o peso de cada componente determinado é relacionado por meio de regressão com variáveis dendrométricas, como descrito na fórmula a seguir:

$$
B=f(P 1, P 2, p 1, p 2, \ldots)
$$

Em que:

$B=$ biomassa em $\mathrm{kg}$ ou ton;

$P=$ variáveis dendrométricas disponíveis, por exemplo, diâmetro $(\mathrm{cm})$ e altura $(\mathrm{m})$;

$p=$ parâmetros do modelo.

As equações alométricas mais comumente utilizadas para a estimativa de biomassa são as propostas por Brown, Gillespie; Lugo (1989), para floresta tropical primária, e por UHL, Buschbacher; Serrão (1988), para o caso de floresta secundária, sendo função do DAP e da altura total do indivíduo:

$$
\begin{gathered}
y=0,44 *\left(D A P^{2} * h\right)^{0,9719} \\
\ln y=-2,17+1,02 \ln (D A P)^{2}+0,39 \ln h \quad \text { (floresta primária) }
\end{gathered}
$$

Em que:

$y=$ biomassa aérea (kg/árvore);

$D A P=$ diâmetro do caule a $1,3 \mathrm{~m}$ de altura do solo $(\mathrm{cm})$;

$h=$ altura total do indivíduo (m). 
A seguir são descritas algumas equações, e seus autores, utilizadas para estimar biomassa acima do solo em regiões tropicais com florestas naturais, a partir de dados de inventário florestal:

$$
\begin{aligned}
& Y=\exp \{-1,996+2,32 * \ln (d)\}-\text { Brown; Gillespie; Lugo (1989) } \\
& Y=10^{(-0,535+\log (B A)\}}-\text { Martinez-Yrizar } \text { et al. }(1992) \\
& Y=42,69-12,800(d)+1,242\left(d^{2}\right)-\text { Brown; Gillespie; Lugo (1989) } \\
& Y=\exp \{-2,134+2,530 * \ln (d)\}-\text { Brown; Gillespie; Lugo (1989) } \\
& Y=2,297-6,95(d)+0,740\left(d^{2}\right)-\text { Brown; Iverson (1992) }
\end{aligned}
$$

Em que:

$Y=$ biomassa por árvore $(\mathrm{kg})$;

$d=$ diâmetro à altura do peito $(\mathrm{cm})$;

$B A=$ área basal $\left(\mathrm{cm}^{2}\right)$.

O objetivo deste trabalho é testar modelos matemáticos, 10 aritméticos e 9 logarítmicos, que visam estimar a biomassa individual aérea em Floresta Ombrófila Densa, e selecionar o melhor deles, com base nos critérios estatísticos mais comumente utilizados na ciência florestal.

\section{METODOLOGIA}

\section{Área de estudo}

As áreas florestais estudadas localizam-se entre os paralelos $27^{\circ} 08^{\prime} 34^{\prime \prime}$ e $27^{\circ} 15^{\prime} 37^{\prime \prime}$ latitude sul, e entre os meridianos $49^{\circ} 11^{\prime} 57^{\prime \prime}$ e $49^{\circ} 17^{\prime} 28^{\prime \prime}$ longitude oeste de Greenwhich, nos municípios de Apiúna e Presidente Nereu, estado de Santa Catarina, compostos pelo baixo, médio e alto vale do Rio Itajaí e compreendendo uma área total de 3.799,2 hectares. A área é composta por seis fazendas: Agrião, Estragão, Itajaí-Mirim, Jundiá, Sabiá e Tranqueira. As fazendas Agrião, Itajaí-Mirim e Sabiá pertencem ao município de Presidente Nereu, enquanto que as fazendas Estragão, Jundiá e Tranqueira pertencem ao município de Apiúna.

\section{Procedimentos metodológicos}

De um inventário florestal, foram coletadas informações de dap em 48 amostras, distribuídas em toda a área de estudo. Desse inventário, foram amostradas, pelo método destrutivo, 213 árvores, nas quais foram determinadas a biomassa total, a cubagem para volume comercial, as densidades básicas, os teores de carbono e as medições das alturas e dos diâmetros. Como o objetivo do trabalho era testar modelos matemáticos para biomassa total, consideramos somente as informações de biomassa total, altura total e DAP.

Para se conhecer a distribuição diamétrica dessas 213 árvores, elaborou-se a tabela 1, que mostra a distribuição de frequência dessas árvores por classes de DAP. A definição do número de classes de diâmetro foi feita utilizando-se a fórmula de Sturges, apresentada e discutida por Angelini; Milone (1993).

$$
\mathrm{N}^{\mathrm{o}} \text { de classes }=1,0+3,3 \log (\mathrm{N})
$$

Em que $\mathrm{N}$ é o número de informações disponíveis.

Como procedimento inicial para possibilitar o ajuste dos modelos, elaborou-se uma matriz de correlação linear simples, composta pelas 19 variáveis independentes listadas na tabela 2, que foram correlacionadas com as variáveis dependentes de peso seco, objetivando conhecer a magnitude da associação entre elas, para que as variáveis com as melhores correlações pudessem ser utilizadas nos modelos testados. 
Tabela 1. Distribuição de frequência em classes de diâmetro para as 213 árvores abatidas.

Table 1. Frequency of diameter by class for the 213 felled trees.

\begin{tabular}{lcc}
\hline Classes diamétricas & Frequência & $(\boldsymbol{\%})$ \\
\hline $7,00 \leq 11,00$ & 20 & 9,40 \\
$11,00 \leq 15,00$ & 59 & 27,70 \\
$15,00 \leq 19,00$ & 36 & 16,90 \\
$19,00 \leq 23,00$ & 28 & 13,14 \\
$23,00 \leq 27,00$ & 30 & 14,10 \\
$27,00 \leq 31,00$ & 11 & 5,16 \\
$31,00 \leq 35,00$ & 13 & 6,10 \\
$35,00 \leq 39,00$ & 11 & 5,16 \\
$39,00 \leq 43,00$ & 3 & 1,40 \\
$43,00 \leq 47,00$ & 2 & 0,94 \\
\hline Total & 213 & 100 \\
\hline
\end{tabular}

Tabela 2. Variáveis independentes utilizadas no processo de seleção dos modelos de biomassa.

Table 2. Independent variables used in the selection process for the models of biomass.

\begin{tabular}{|c|c|c|c|c|c|}
\hline $\mathbf{A}$ & B & $\mathrm{C}$ & D & $\mathbf{E}$ & $\mathbf{F}$ \\
\hline dap & 1/dap & lndap & $\ln 1 /$ dap & dapht & lndht \\
\hline ht & $\begin{array}{c}\text { 1/ht } \\
\text { dap }^{2} \\
\text { dap }^{3} \\
\mathrm{ht}^{2}\end{array}$ & $\operatorname{lnht}$ & $\begin{array}{l}\ln 1 / \mathrm{ht} \\
\operatorname{lndap^{2}} \\
\operatorname{lnht}^{2}\end{array}$ & $\begin{array}{l}\text { dap }^{2} h t \\
h t^{2} \text { dap }\end{array}$ & 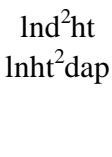 \\
\hline
\end{tabular}

A: variáveis puras; B: inversos e potências; C: logaritmizadas; D: inversos e potências logaritmizadas; E: produtos cruzados; F: produtos cruzados logaritmizados; dap: diâmetro à altura do peito; ht: altura total; ln: logaritmo neperiano.

Após a escolha das variáveis, foram realizados os ajustes de modelos para estimar a biomassa total em $90 \%$ do total de árvores amostradas (192), utilizando os $10 \%$ restantes (21) para fazer a validação do modelo selecionado.

A escolha dessas 21 árvores foi realizada observando-se a frequência de árvores por classe, sendo que, da primeira para a décima classe, retirou-se sucessivamente a seguinte quantidade de árvores: $2,5,3,3,3,1,1,1,1$ e 1 . Nas classes em que foi retirada somente uma árvore, escolheu-se a do centro, e nas demais classes retiraram-se árvores equidistantes umas das outras.

Para a estimativa de biomassa total seca por árvore, foram testados 10 modelos apresentados na forma aritmética e 09 modelos apresentados na forma logarítmica, por meio de regressão linear simples e múltipla. Esses modelos são apresentados na tabela 3.

$\mathrm{Na}$ escolha da melhor equação ajustada, foram adotadas as seguintes estatísticas: Coeficiente de Determinação Ajustado ( $\mathrm{R}^{2}$ aj), Erro Padrão da Estimativa em Percentagem (Syx\%) e Análise Gráfica dos Resíduos, sendo utilizado o Fator de Correção de Meyer para a correção da discrepância logarítmica nos modelos em que a variável dependente sofreu transformação logarítmica.

Além disso, calculou-se a Amplitude Absoluta dos Resíduos (AR), obtida pela diferença entre o maior e o menor resíduo gerado pela equação. Essa estatística foi empregada apenas para situações em que não foi possível a escolha da melhor equação pelas estatísticas anteriores e pela análise de resíduos, por apresentarem comportamento bem próximos uns dos outros. Esse critério determina que a equação que apresentar menor amplitude de resíduos é considerada melhor.

\section{RESULTADOS E DISCUSSÃO}

Na tabela 4, observa-se que a variável dependente biomassa total (BT) apresentou correlação mais alta com as variáveis independentes dap, $\mathrm{dap}^{2}, \mathrm{dap}^{3}, \mathrm{dap}^{2} \mathrm{ht}$ e $\operatorname{lnht}^{2} \mathrm{dap}$. Em sua forma logarítmica, essa variável apresentou alta correlação com as variáveis dependentes dap, 1/dap, dapht, lndap, ln1/dap, $\operatorname{lndap}^{2}, \operatorname{lndap}^{3}$ e $\operatorname{lnht}^{2}$ dap. Essas análises informam que, pela alta correlação entre as variáveis dependentes e a variável independente "dap", tanto em sua forma pura como combinada, esta terá que obrigatoriamente fazer parte dos modelos que visam à estimativa dessas variáveis. 
Tabela 3. Modelos testados para estimativa de biomassa total seca.

Table 3. Models tested to estimate the total biomass dry.

\begin{tabular}{lll}
\hline $\mathbf{N}^{\mathbf{0}}$ & \multicolumn{1}{c}{ Aritméticos } & \multicolumn{1}{c}{ Logarítmicos } \\
\hline 1 & $B T=b_{0}+b_{1} d a p$ & $\ln B T=b_{0}+b_{1} \ln d a p$ \\
2 & $B T=b_{0}+b_{1} d a p^{2}$ & $\ln B T=b_{0}+b_{1} \ln d a p^{2}$ \\
3 & $B T=b_{0}+b_{1} d a p+b_{2} d a p^{2}+b_{3} d a p^{3}$ & $\ln B T=b_{0}+b_{1} \ln d a p+b_{2} \ln h t^{2} d a p$ \\
4 & $B T=b_{0}+b_{1} d a p+b_{2} d a p h t$ & $\ln B T=b_{0}+b_{1} \ln h t^{2} d a p$ \\
5 & $B T=b_{0}+b_{1} d a p^{2}+b_{2} d a p^{2} h t$ & $\ln B T=b_{0}+b_{1} \ln d a p^{2}+b_{2} \ln h t^{2} d a p$ \\
6 & $B T=b_{0}+b_{1} d a p^{2}+b_{2} d a p^{2} h t+b_{3} \ln h t^{2} d a p$ & $\ln B T=b_{0}+b_{1} d a p h t$ \\
7 & $B T=b_{0}+b_{1} d a p^{2} h t$ & $\ln B T=b_{0}+b_{1} \ln d a p+b_{2} d a p h t$ \\
8 & $B T=b_{0}+b_{1} d a p^{2}+b_{2} h t^{2}+b_{3} d a p^{2} h t$ & $\ln B T=b_{0}+b_{1} \ln d a p+b_{2} \ln h t$ \\
9 & $* B T=b_{0}+d a p^{2} h t+h t^{2} h c$ & $* \ln B T=b_{0}+b_{1} \ln d a p^{2}+b_{2} \ln (1 / h t)$ \\
10 & $B T=b_{0}+(d a p h t)^{b 1}$ &
\end{tabular}

BT: peso seco (kg); dap: diâmetro à altura do peito $(\mathrm{cm})$; ht: altura total $(\mathrm{m})$; ln: logaritmo neperiano; $\mathrm{b}_{\mathrm{i}}$ : coeficientes dos modelos. * equações ajustadas pelo método "Forward".

A alta correlação entre a variável biomassa total e o dap, mencionada anteriormente, pode também ser comprovada na figura 1 . Outra informação que pode ser extraída desta figura é a existência de pequena heterocedasticidade à medida que os valores de dap aumentam. Este fato pode ser explicado tendo em vista que estes dados são originados de floresta de formação natural, com uma alta diversidade de espécies, e como tal, apresentando indivíduos com as mais diversas formas e alturas.

Tabela 4. Coeficientes de correlação de Pearson entre as variáveis dependentes de peso seco e variáveis independentes.

Table 4. Coefficients of the Pearson correlation between variables dependent on dry weight and independent variables.

\begin{tabular}{|c|c|c|c|c|c|}
\hline VI & BT & $\operatorname{lnBT}$ & VI & BT & $\operatorname{lnBT}$ \\
\hline dap & 0,93 & 0,95 & lndap & 0,88 & 0,97 \\
\hline ht & 0,64 & 0,71 & lnht & 0,63 & 0,73 \\
\hline 1/dap & $-0,8$ & $-0,95$ & $\ln 1 / \mathrm{ht}$ & $-0,88$ & $-0,97$ \\
\hline $1 / \mathrm{ht}$ & $-0,61$ & $-0,72$ & $\ln 1 / \mathrm{ht}$ & $-0,63$ & $-0,73$ \\
\hline $\mathrm{dap}^{2}$ & 0,95 & 0,89 & lndap ${ }^{2}$ & 0,88 & 0,97 \\
\hline $\mathrm{dap}^{3}$ & 0,93 & 0,82 & lndap ${ }^{3}$ & 0,88 & 0,97 \\
\hline $\mathrm{ht}^{2}$ & 0,62 & 0,67 & $\operatorname{lnht} t^{2}$ & 0,63 & 0,73 \\
\hline dapht & 0,93 & 0,92 & lndapht & 0,8 & 0,86 \\
\hline hcht & 0,4 & 0,47 & lnhcht & 0,49 & 0,57 \\
\hline $\mathrm{dap}^{2} \mathrm{ht}$ & 0,96 & 0,89 & lndap ${ }^{2} h t$ & 0,8 & 0,86 \\
\hline$h t^{2}$ dap & 0,86 & 0,84 & $\operatorname{lnht^{2}} d a p$ & 0,94 & 0,95 \\
\hline
\end{tabular}

VI: variáveis independentes; BT: biomassa total (kg); lnBT: logaritmo neperiano da biomassa total (base "e"); dap: diâmetro à altura do peito $(\mathrm{cm})$; ht: altura total $(\mathrm{m}) ; \ln$ : logaritmo neperiano.

As tabelas 5 e 6 apresentam as equações ajustadas nas formas aritméticas e logarítmicas, respectivamente, bem como os seus coeficientes, as estatísticas calculadas para a verificação da qualidade desses ajustes e a amplitude residual para os modelos pré-selecionados. 


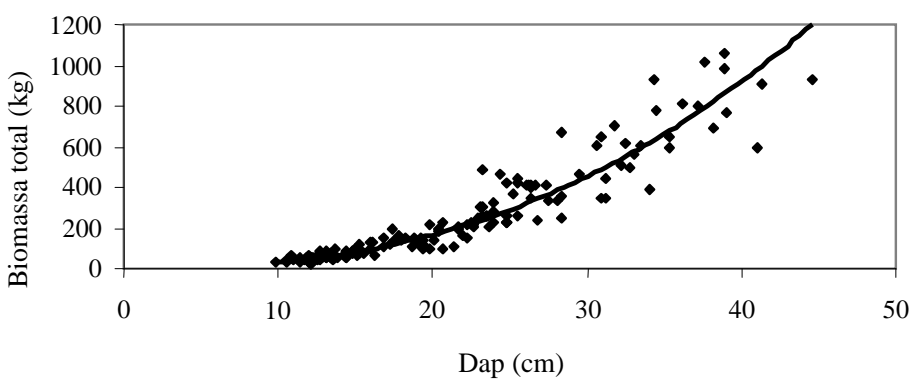

Figura 1. Diagrama de dispersão de biomassa total em relação ao dap.

Figure 1. Diagram of dispersal of total biomass in relation to the diameter at breast height.

Tabela 5. Equações ajustadas na forma aritmética com suas respectivas estatísticas de ajuste, precisão e a amplitude residual para a estimativa de biomassa total.

Table 5. Equations adjusted in arithmetic form with their respective statistical adjustment, accuracy and range residual for the estimated total biomass.

\begin{tabular}{|c|c|c|c|c|}
\hline$\overline{N^{0}}$ & Equações & $R^{2} \mathbf{a j}$ & Syx\% & $\mathbf{A R}$ \\
\hline 1 & $B T=-343,16623+28,24845$ dap & 0,86 & 36,12 & \\
\hline 2 & $B T=-44,44802+0,57853 d a p^{2}$ & 0,89 & 31,36 & $119,09 *$ \\
\hline 3 & $B T=240,02741-39,16940 d a p+2,19857 d a p^{2}-0,02049 d a p^{3}$ & 0,89 & 31,12 & 188,99 \\
\hline 4 & $B T=-268,76681+15,68512 d a p+0,60685 d a p h t$ & 0,88 & 32,75 & \\
\hline 5 & $B T=-13,42600+0,14563 d a p^{2}+0,02376 d a p^{2} h t$ & 0,92 & 25,90 & 91,50 \\
\hline 6 & $B T=-2,01269+0,15677 d a p^{2}+0,02454 d a p^{2} h t-0,20696 \ln h t^{2} d a p$ & 0,93 & 25,97 & 89,63 \\
\hline 7 & $B T=1,13617+0,03128 d a p^{2} h t$ & 0,92 & 26,46 & 93,52 \\
\hline 8 & $B T=25,87071+0,02909 d a p^{2}-0,21382 h t^{2}+0,03189 d a p^{2} h t$ & 0,93 & 25,58 & 98,01 \\
\hline 9 & $B T=13,6215+0,03277 d a p^{2} h t-0,01350 h t^{2} h c$ & 0,93 & 25,47 & 137,97 \\
\hline 10 & $B T=0,09145(\text { dapht })^{1,383}$ & 0,85 & 36,82 & \\
\hline
\end{tabular}

* A amplitude dos resíduos somente foi calculada para as equações pré-selecionadas.

Em uma análise inicial dos resultados, observa-se que as equações apresentaram bons resultados com base no coeficiente de determinação ajustado $\left(R^{2}\right.$ aj), sendo que as equações aritméticas apresentaram melhores ajustes para essa estatística que as logarítmicas. Com exceção das equações logarítmicas 4 e 6 , que apresentaram valores de 0,62 e 0,35, respectivamente, para o $\mathrm{R}^{2} \mathrm{aj}$, as demais equações apresentaram boa qualidade de ajuste, com valores que variaram de 0,86 a 0,93 , indicando a variação percentual da variável dependente, que é explicada pelas variáveis independentes. O melhor ajuste para essa estatística foi observado nas equações aritméticas 6,8 e 9, que apresentaram um valor de 0,93 , enquanto que a equação 6 logarítmica apresentou o pior ajuste, com um valor de 0,35.

Com relação ao erro padrão da estimativa, os resultados indicam um desempenho semelhante para essa estatística entre as equações aritméticas e logarítmicas, com exceção das equações logarítmicas 4 e 6 que, da mesma forma como ocorreu para o $\mathrm{R}_{\mathrm{aj}}^{2}$, apresentaram os piores ajustes, com valores de $65,50 \%$ e $87,33 \%$, respectivamente.

Observando os valores da tabela 6, apesar de haver uma alta correlação linear entre as variáveis $\operatorname{lnBT}$ e $\operatorname{lnht}^{2} \mathrm{~d}$ e as variáveis $\operatorname{lnBT}$ e dapht, com $\mathrm{r}=0,95$ e 0,92 , respectivamente, quando colocadas no mesmo modelo, 4 e 6, após o ajuste, o Syx\% da equação apresentou-se muito alto, com valores de $65,50 \%$ e $87,33 \%$. Com exceção dessas duas equações, as demais, tanto nas formas aritméticas quanto logarítmicas, tiveram uma variação entre $25,47 \%$ e $36,82 \%$. 
Tabela 6. Equações ajustadas na forma logarítmica com suas respectivas estatísticas de ajuste, precisão e a amplitude residual para a estimativa de biomassa total.

Table 6. Equations adjusted in logarithmic form with their respective statistical adjustment, accuracy and range residual for the estimated total biomass.

\begin{tabular}{llcccc}
\hline \multicolumn{1}{c}{ Equações } & $\mathbf{R}^{\mathbf{2}} \mathbf{a j}$ & $\mathbf{S y x} \%$ & $\mathbf{F C M e y e r}$ & $\mathbf{A R}$ \\
\hline 1 & $\ln B T-2,22923+2,45462 \ln d a p$ & 0,88 & 32,72 & 1,03450 & \\
2 & $\ln B T=-2,22923+1,22731 \ln d a p^{2}$ & 0,88 & 32,72 & 1,03450 & \\
3 & $\ln B T=-0,77949+1,77175 \ln d a p+0,00516 \ln h t^{2} d a p$ & 0,87 & 35,48 & 1,03246 & \\
4 & $\ln B T=3,07231+0,01776 \ln h t^{2} d a p$ & 0,62 & 65,50 & 1,04667 & \\
5 & $\ln B T=-0,77949+0,88587 \ln d a p^{2}+0,00516 \ln h t^{2} d a p$ & 0,87 & 35,48 & 1,03246 & \\
6 & $\ln B T=3,557106+0,00483 d a p h t$ & 0,35 & 87,33 & 1,07746 & \\
7 & $\ln B T=-1,03353+1,92706 \ln d a p+0,00118 d a p h t$ & 0,89 & 32,06 & 1,03059 & \\
8 & $\ln B T=-2,44886+2,05684 \ln d a p+0,55255 \ln h t$ & 0,91 & 26,37 & 1,02852 & $78,25^{*}$ \\
9 & $\ln B T=-2,96459+1,10152 \ln d a p^{2}-0,56933 \ln (1 / h t)$ & 0,91 & 26,37 & 1,02852 & 78,25 \\
\hline
\end{tabular}

* A amplitude dos resíduos somente foi calculada para as equações pré-selecionadas.

As duas equações ajustadas pelo método Forward apresentaram os melhores resultados dentro de seus conjuntos de equações (aritméticas e logarítmicas), com valores de 0,93 e 0,91 para o $\mathrm{R}^{2}$ aj e 25,47\% e $26,37 \%$ para o Syx \%.

Como quesito fundamental na escolha de uma equação de crescimento ou de produção, realizouse a análise gráfica dos resíduos, para verificação da distribuição dos resíduos da regressão de cada equação, uma vez que erros de tendência podem estar ocorrendo em alguma amplitude de classe de uma ou mais variáveis independentes sem ser detectados pelas estatísticas que medem a exatidão. Esses gráficos são mostrados nas figuras 2 e 3 .

Nos gráficos de resíduos das equações aritméticas, observam-se dois tipos de comportamento nas estimativas de biomassa total para diâmetros abaixo e acima de $20 \mathrm{~cm}$. Para diâmetros abaixo de $20 \mathrm{~cm}$, com exceção das equações 1 e 4, as estimativas são mais exatas, pois a amplitude dos resíduos é menor, apresentando uma variância homogênea. Para diâmetros acima de $20 \mathrm{~cm}$, as estimativas são menos exatas, pois apresentam uma amplitude maior dos resíduos, porém apresentando também uma variância homogênea. Nas equações 1 e 4, observa-se nitidamente que, para valores com diâmetros abaixo de $20 \mathrm{~cm}$, há uma tendência de subestimativas passando para superestimativas até este valor.

Nos modelos logarítmicos, os gráficos dos resíduos se apresentaram semelhantes aos aritméticos para diâmetros menores que $20 \mathrm{~cm}$, ou seja, pequena amplitude dos resíduos e variância homogênea. Para diâmetros maiores de $20 \mathrm{~cm}$, com exceção das equações 8 e 9 , que apresentaram uma variância homogênea, as demais apresentaram uma tendência a superestimativas à medida que os valores de dap aumentam. Com base nessas análises, as equações pré-selecionados foram: 2, 3, 5, 6, 7, 8 e 9 aritméticas, e 8 e 9 logarítmicas.

Como último critério para a escolha da melhor equação, observa-se, pela amplitude residual absoluta, que os modelos logarítmicos 8 e 9 apresentaram os menores valores, ou seja, 78,25. Dessa forma, a equação 8 foi a selecionada, pois, sendo mais simplificada, facilita os cálculos das estimativas de biomassa total para as árvores inventariadas.

Para fins de validação das equações, estimou-se, pela equação selecionada, a biomassa total das 21 árvores separadas do conjunto inicial de árvores. Esse valor resultante foi comparado com o valor real medido por meio do teste de Qui-quadrado $\left(\chi^{2}\right)$. O valor resultante do teste foi $\chi^{2}=13,25$, indicando que não existem diferenças estatisticamente significativas entre as biomassas reais e as biomassas estimadas pela equação selecionada, ao nível de $99 \%$ de probabilidade.

Com o objetivo de se obter uma informação adicional relacionada à qualidade dessa equação, comparou-se o somatório da biomassa real e o somatório da biomassa estimada nas 21 árvores separadas para validação. Enquanto que o somatório da biomassa real para essas árvores foi de 73,70 t, para as árvores estimadas foi de 69,88 t, significando uma diferença de apenas 5,18\%. 

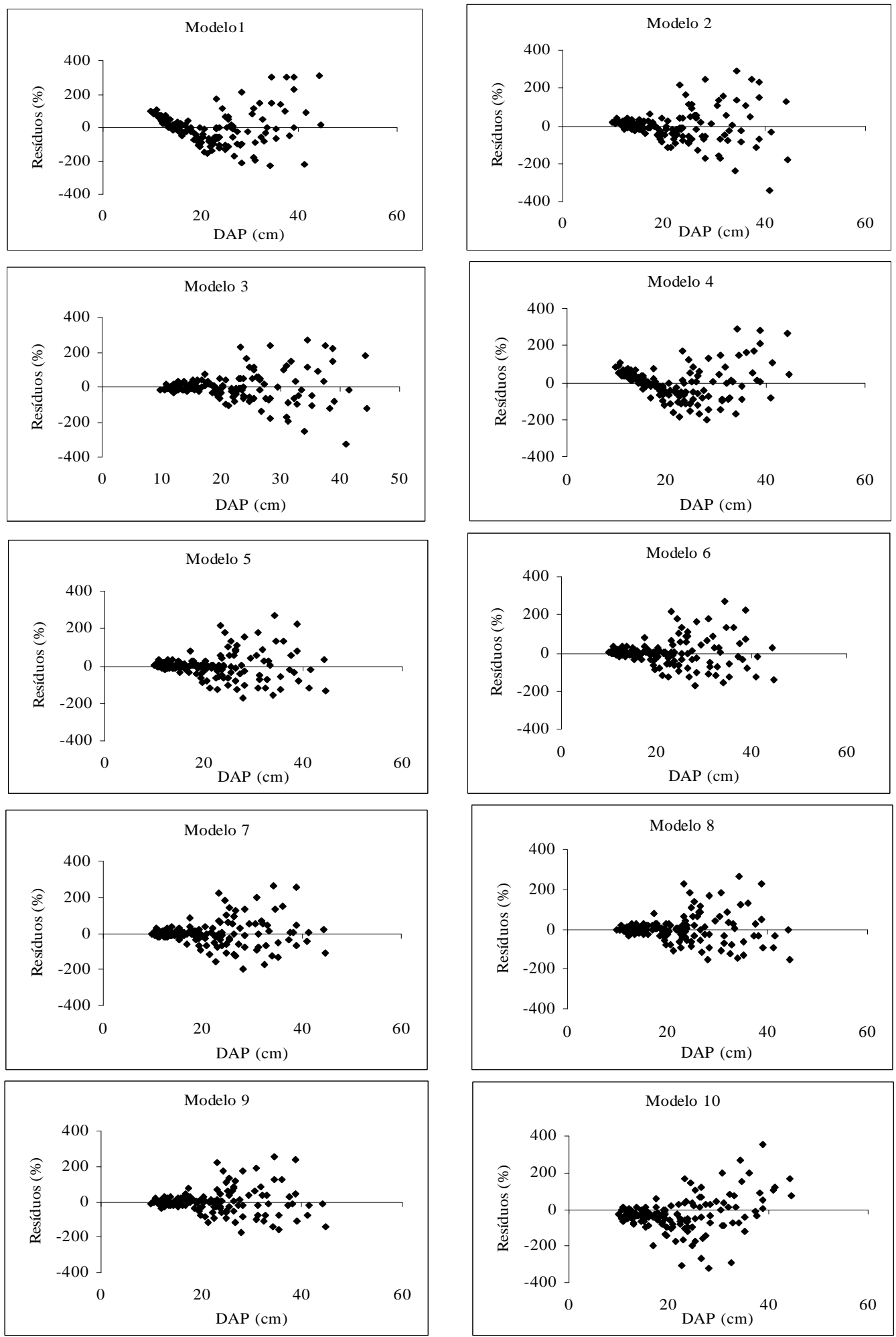

Figura 2. Distribuição gráfica dos resíduos de biomassa total em função do dap para os modelos aritméticos.

Figure 2. Residual graphics distribution of total biomass depending for the diameter at breast height on the models arithmetic. 

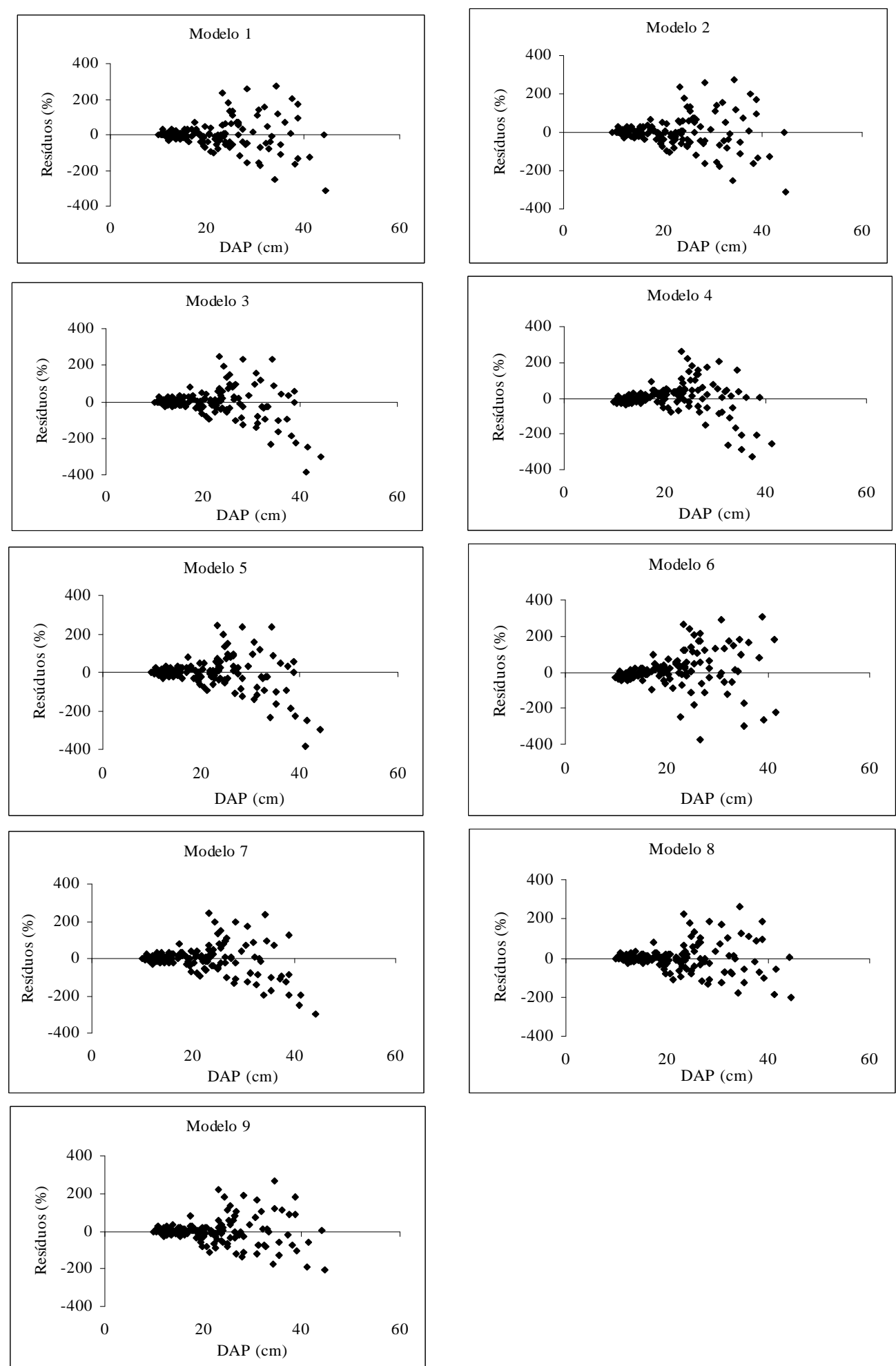

Figura 3. Distribuição gráfica dos resíduos de biomassa total em função do dap para os modelos logarítmicos.

Figure 3. Residual graphics distribution of total biomass depending for the diameter at breast height on the models logarithmic. 


\section{CONCLUSÕES E RECOMENDAÇÕES}

- Os modelos aritméticos e logarítmicos testados para se estimar biomassa aérea, de um modo geral apresentaram bons ajustes, com várias equações apresentando comportamentos bem semelhantes.

- O critério Amplitude Residual (AR), apesar de não ser comumente utilizado em trabalhos de ajuste de modelos matemáticos na ciência florestal, neste trabalho mostrou-se muito útil, uma vez que serviu como critério de desempate para a escolha do melhor modelo.

- Em função da qualidade e semelhança nos ajustes para os modelos aritméticos e logarítmicos, em estudos de biomassa essas duas formas de modelos terão sempre que ser testadas, caso contrário corre-se o risco de que não sejam testados modelos com grande potencial de ajuste.

- Mesmo apresentando um erro de $26,37 \%$, tendo em vista a variabilidade natural existente na estrutura da vegetação e na forma dos troncos das espécies lenhosas desse tipo de floresta, a equação selecionada é recomendada para estimar biomassa total individual em Floresta Ombrófila Densa, podendo ser utilizada em planos de manejo, pesquisas e projetos de biomassa e sequestro de carbono.

\section{REFERÊNCIAS}

ANGELINI, F.; MILONE, G. Estatística geral. São Paulo, 1993. 206 p.

BROWN, S.; GILLESPIE, A. J. R.; LUGO, A. E. Biomass estimation methods for tropical forests with applications to forest inventory data. Forest Science, Lawrence, v. 35, p. 881-902, 1989.

BROWN, S.; IVERSON, L. R. Biomass estimates for tropical forests. World Resources Review, [S.1.], n. 4, p. 366-384, 1992.

BROWN, S. L.; SCHOEDER, P.; KERN, J. S. Spatial distribution of biomass in forests of the eastern USA. Forest Ecology and Management, Amsterdam, v. 123, n. 1-3, p. 81-90, 1999.

DIXON, R. K.; HOUGHTON, R. A.; SOLOMON, A. M.; TREXLER, M. C.; WISNIEVSKI, J. Carbon Pools and Flux of Global Forest Ecossystems. Science, Washington, D. C., v. 263, p. 185-190, 1994.

FAN, S.; GLOOR, M.; MAHLMAN, J.; PACALA, S.; SARMIENTO, J.; TAKAHASHI, T. A large terrestrial carbon sink in north America implied by atmospheric and oceanic carbon dioxide models. Science, Washington, D. C., n. 282, p. 386-387, 1998.

HIGUCHI, N.; CARVALHO JÚNIOR, J. A. Fitomassa e conteúdo de carbono de espécies arbóreas da Amazônia. In: SEMINÁRIO EMISSÃO X SEQÜESTRO DE $\mathrm{CO}_{2}$ - uma nova oportunidade de negócios para o Brasil, 1994, Rio de Janeiro. Anais... Rio de Janeiro: CVRD, 1994. p. 125-145.

KOEHLER, H. S.; WATZLAWICK, L. F.; KIRCHNER, F. F. Fontes e níveis de erros nas estimativas do potencial de fixação de carbono. In: SANQUETA, C. R. et al. (Eds.). As florestas e o carbono. Curitiba, 2002. p. 251-264.

SANQUETTA, C. R. Métodos de determinação de biomassa florestal. In: SANQUETTA, C.R. et al. (Eds.). As florestas e o carbono. Curitiba, 2002, p. 119-140.

MARTINEZ-YRIZAR, SARUKHAN, A. J.; PEREZ-JIMENEZ, A.; RINCÓN, E.; MAASS, J. M.; SOLIMAGALLANES, A.; CERVANTES, L. Aboveground phytomass of a tropical deciduous forest on the coast of Jalisco, Mexico. Journal of Tropical Ecology, Cambridge, v. 8, p. 87-96, 1992.

SEDJO, R. A. Temperate Forest ecosystem in the global carbon cycle. Ambio, Stockholm, v. 21, p. $274-$ 277, 1992.

SOMOGYI, Z.; CIENCIALA, E.; MÄKIPÄÄ, MUUKKONEN, P.; LEHTONEN A.; WEISS, P. Indirect methods of large forest biomass estimation. Europe Journal Forest Research, [S.1.], . February (2006).

UHL, C.; BUSCHBACHER, R.; SERRÃO, E. A. S. Abandoned pastures in Eastern Amazonia. I. Patterns of plant succession. The Journal of Ecology, Oxford, v. 76, p. 663-681, 1988. 